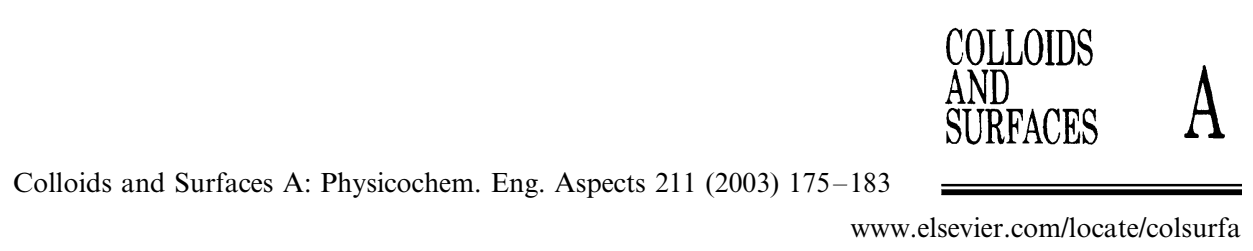

www.elsevier.com/locate/colsurfa

\title{
Diffusioosmosis of nonelectrolyte solutions in a fibrous medium
}

\author{
Yeu K. Wei, Huan J. Keh* \\ Department of Chemical Engineering, National Taiwan University, Taipei 106-17, Taiwan, ROC
}

Received 9 October 2002; accepted 26 March 2003

\begin{abstract}
The steady diffusioosmotic flow of a fluid solution of an uncharged solute in the fibrous porous medium constructed by a homogeneous array of parallel circular cylinders is analytically studied. The imposed solute concentration gradient is constant and can be oriented arbitrarily with respect to the axes of the cylinders. The range of the interaction between the solute molecules and the cylinder surfaces is assumed to be small relative to the radius of the cylinders and to the gap thickness between two neighboring cylinders, but the effect of polarization of the mobile solute in the thin diffuse layer surrounding each cylinder caused by the strong adsorption of the solute is taken into account. Through the use of a unit cell model, the appropriate equations of conservation of mass and momentum are solved for each cell, in which a cylinder is envisaged to be surrounded by a coaxial shell of the fluid solution. Analytical expressions for the diffusioosmotic velocity of the bulk fluid as functions of the porosity of the ordered array of cylinders are obtained for various cases. Comparisons of the results of the cell model with different conditions at the outer boundary of the cell are made. In the limit of maximum porosity, these results can be interpreted as the diffusiophoretic velocity of an isolated circular cylinder caused by the imposed solute concentration gradient.
\end{abstract}

(C) 2003 Elsevier Science B.V. All rights reserved.

Keywords: Diffusioosmosis; Diffusiophoresis; Circular cylinder; Polarized diffuse layer; Unit cell model

\section{Introduction}

The flow behavior of fluids in porous media is of much fundamental and practical interest. In general, driving forces for the fluid transport through micropores include dynamic pressure differences between the two ends of the pores (convection),

\footnotetext{
* Corresponding author. Tel.: +886-2-23635462; fax: +8862-23623040.

E-mail address: huan@ccms.ntu.edu.tw (H.J. Keh).
}

concentration differences of a solute between the two bulk solutions outside the pores which do not permit the passage of the solute or permit its passage but exerts more resistance on it than on the solvent molecules (osmosis), and tangential electric fields that interact with the electric double layer adjacent to a charged pore wall (electroosmosis). Problems of fluid flow induced by these well-known driving forces were treated extensively in the past.

Another driving force for the flow of fluid solutions in micropores, which has commanded 
less attention, involves tangential concentration gradients of a solute that is freely penetrable through the pore and interacts with the pore wall. The fluid motion associated with this mechanism, which is termed 'diffusioosmosis', has been discussed for solutions of either ionic or nonionic solutes near a plane wall [1-3] and inside a straight capillary [4-7]. For an electrolyte solution in contact with a charged wall, the solute-wall interaction is electrostatic in nature. On the other hand, in a solution of uncharged solute, the solute molecules interact with the wall through the van der Waals/dipole forces. For a solution of nonionic solute with a constant concentration gradient $\nabla n_{\infty}$ along a plane wall, the diffusioosmotic velocity outside the solute-wall interaction layer (diffuse layer) can be expressed as [2]

$V=-\frac{k T}{\eta} L^{*} K \nabla n_{\infty}$

Here, $L^{*}$ is a positive characteristic length for the solute-wall interaction (of order $1 \mathrm{~nm}$ ), $K$ is the Gibbs adsorption length characterizing the strength of the adsorption of the molecular solute ( $K$ and $L^{*}$ are defined by Eqs. (6c) and (6d), and $K$ is positive if the interaction force between the solute and the wall is attractive), $\eta$ is the fluid viscosity, $k$ is Boltzmann's constant, and $T$ is the absolute temperature. The negative sign in Eq. (1) implies that the fluid solution flows toward the side of low solute concentration when the solutewall interaction force is attractive.

The analytical solution for the diffusioosmotic velocity of a bulk fluid solution parallel to a plane wall given by Eq. (1) can also be applied to the corresponding flow in straight capillaries when the thickness of the diffuse layer adjacent to the capillary wall is small compared with the capillary radius. However, the capillary model of porous media is not a realistic model for either granular or fibrous systems, for it does not allow for the convergence and divergence of flow channels. For diffusioosmotic flow within beds of particles, fiber matrices, or microporous membranes, it is usually necessary to account for the effects of pore geometry, tortuosity, etc. To avoid the difficulty of the complex geometry appearing in beds of particles, unit cell models were often employed to predict these effects on the relative motions between a granular bed and the bulk fluid. These models involve the concept that a bed of identical particles can be divided into a number of identical cells, one particle occupying each cell at its center. The boundary value problem for multiple particles is thus reduced to the consideration of the behavior of a single particle and its bounding envelope. The most acceptable of these models with various boundary conditions at the outer (virtual) surface of a cell are the 'free-surface' model of Happel [8,9] and 'zero-vorticity' model of Kuwabara [10]. In the past, the unit cell models were used by many researchers to predict various transport properties such as the mean sedimentation rate [11-13] and electrophoretic mobility [14$21]$ in a suspension of charged spherical particles as well as the electroosmotic mobility of an electrolyte solution in a fibrous porous medium [22-25]. Recently, using the cell models, the present authors derived analytical expressions for the mean diffusiophoretic velocity of a swarm of colloidal spheres [26], which can also apply for the diffusioosmotic velocity within a fixed bed of spherical particles.

In this work, the Happel and Kuwabara cell models are used to obtain analytical expressions for the diffusioosmotic velocity of a solution of uncharged solute with a uniformly prescribed and arbitrarily oriented concentration gradient within a homogeneous array of parallel circular cylinders. The thickness of the solute-wall interaction layers is assumed to be small relative to the radius of cylinders and to the gap width between two neighboring cylinders, but the polarization effect of the mobile solute in the thin diffuse layers caused by the strong adsorption of the solute is allowed. The analytical solutions in closed form obtained with the cell models enable the diffusioosmotic velocity of the bulk fluid to be predicted as functions of the porosity of the fiber matrix for various cases. The information provided by this work may prove relevant in understanding the chemotactic flow of fluids and transport of particles in physiological media. 


\section{Analysis}

We consider the diffusioosmosis of a fluid solution of a nonionic solute through a uniform array of parallel, identical, long circular cylinders at the steady state. The solute molecules can diffuse freely in this fibrous porous medium, so there exists no regular osmotic flow of the solvent. The thin diffuse layers (solute-wall interaction layers) surrounding the cylinders can be polarized, but they do not overlap with one another. The uniformly imposed solute concentration gradient $\nabla n_{\infty}$ (which is the concentration gradient existing in the absence of the cylinders) can be regarded as a combination of the transversal and longitudinal components with respect to the orientation of the cylinders,

$\nabla n_{\infty}=E_{\mathrm{t}} \mathbf{e}_{x}+E_{1} \mathbf{e}_{z}$

where $\mathbf{e}_{x}$ and $\mathbf{e}_{z}$ are the unit vectors in the directions perpendicular and parallel, respectively, to the axes of the cylinders. Then, the problem can be divided into two due to the linearity and they will be separately solved. The total diffusioosmotic velocity of the bulk fluid can be obtained by the vectorial addition of the two-component results.

First, we consider the diffusioosmotic motion of the fluid solution due to the transverse component of the solute gradient. The bulk diffusioosmotic velocity of the solution generated by the gradient $E_{\mathrm{t}} \mathbf{e}_{x}$ can be written as $-U_{\mathrm{t}} \mathbf{e}_{x}$ (which is the velocity of the uniform flow in the bulk fluid beyond the

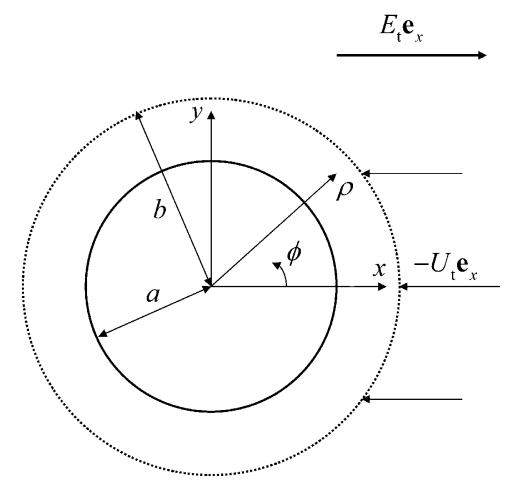

Fig. 1. Geometrical sketch for the transverse diffusioosmosis of a fluid solution around a circular cylinder in a coaxial cylindrical cell. fibrous mat). As shown in Fig. 1, we employ a unit cell model in which each cylinder of radius $a$ is surrounded by a coaxial circular cylindrical shell of the fluid solution having an outer radius of $b$ such that the fluid/cell volume ratio is equal to the porosity $1-\varphi$ of the fiber matrix; viz., $\varphi=(a / b)^{2}$. The origin of the polar coordinate system $(\rho, \phi)$ is taken at the axis of the cylinder and the polar axis $\phi=0$ points toward the positive $x$ direction. Obviously, the two-dimensional problem for each cell is symmetric about the $x$ axis. Our objective is to determine the bulk velocity of the solution induced by the diffusioosmotic driving force.

The Peclet number $\left(U a / D_{0}\right.$, where $D_{0}$ is the diffusion coefficient of the solute molecules in the fluid) of this problem is assumed to be small. Hence, the equation of continuity governing the solute concentration distribution $n(\rho, \phi)$ for the fluid solution is the Laplace equation

$\nabla^{2} n=0$.

The boundary condition for the solute concentration at the cylinder surface (or, more precisely, at the outer limit of the thin polarized interfacial layer) requires that [27-29]

$\rho=a: \quad \frac{\partial n}{\partial \rho}=-\beta \frac{1}{\rho^{2}} \frac{\partial^{2} n}{\partial \phi^{2}}$,

where the relaxation coefficient

$\beta=(1+v \mathrm{Pe}) K$,

with

$$
\begin{aligned}
\mathrm{Pe}= & \frac{k T}{\eta D_{0}} L^{*} K n_{0}, \\
v= & \left(L^{*} K^{2}\right)^{-1} \int_{0}^{\infty}\left\{\int _ { y } ^ { \infty } \left[\exp \left(\frac{-\Phi(y)}{k T}\right)\right.\right. \\
& -1] \mathrm{d} y\}^{2} \mathrm{~d} y, \\
K= & \int_{0}^{\infty}\left[\exp \left(\frac{-\Phi(y)}{k T}\right)-1\right] \mathrm{d} y, \\
L^{*} & =K^{-1} \int_{0}^{\infty} y\left[\exp \left(\frac{-\Phi(y)}{k T}\right)-1\right] \mathrm{d} y .
\end{aligned}
$$

In Eqs. (6a), (6b), (6c) and (6d), $\Phi$ represents the potential energy resulting from the interaction 
between a single solute molecule and the cylinder surface $(\Phi$ is positive if the interaction force is repulsive and negative if it is attractive), $y$ is the normal distance measured from the cylinder surface to the fluid phase, $n_{0}$ is the prescribed solute concentration measured at the position of the axis of the cylinder but in the absence of the cylinder, and it has been assumed that $a E_{\mathrm{t}} / n_{0} \ll 1$. The dimensionless parameter $v$ depends primarily on the shape of the function $\Phi$ rather than on its magnitude, and a typical value for it is $1 / 2$. The adsorption length $K$ equals the ratio of the Gibbs excess concentration of the solute in the adsorption layer to the solute concentration in the bulk solution at equilibrium, and it can be measured independently. There does not seem to be any means of quantitatively determining the length $L^{*}$, other than to say that it is positive and comparable to the size of the solute molecule. The physical meaning of Eq. (4) is that the net tangential solute flux along the cylinder surface must be balanced by the normal solute flux occurred just beyond the diffuse layer to prevent accumulation of the solute. The relaxation coefficient $\beta$ represents the ratio of apparent surface-to-bulk diffusion coefficients [28].

At the outer (virtual) surface of the cell, the local solute concentration gradient is in accord with the uniformly applied gradient $E_{\mathrm{t}} \mathbf{e}_{x}$. Thus,

$\rho=b: \quad \frac{\partial n}{\partial \rho}=E_{\mathrm{t}} \cos \phi$.

The solution to Eqs. (3), (4) and (7) is

$n=n_{0}+A\left[1+\frac{\beta}{a}+\left(1-\frac{\beta}{a}\right) \frac{a^{2}}{\rho^{2}}\right] E_{\mathrm{t}} \rho \cos \phi$,

where

$A=\left[1+\frac{\beta}{a}-\left(1-\frac{\beta}{a}\right) \varphi\right]^{-1}$

and $\varphi=(a / b)^{2}$.

An alternative for the boundary condition of the solute concentration at the virtual surface $\rho=b$ may be taken as the distribution giving rise to the gradient $E_{\mathrm{t}} \mathbf{e}_{x}$ in the cell when the cylinder does not exist. In this case, Eq. (7) becomes $\rho=b: \quad n=n_{0}+E_{\mathrm{t}} \rho \cos \phi$.

The solution of the governing Eq. (3) subject to the boundary conditions Eqs. (4) and (10) is also given by the form of Eq. (8), but with the parameter $A$ defined as

$A=\left[1+\frac{\beta}{a}+\left(1-\frac{\beta}{a}\right) \varphi\right]^{-1}$.

For the special case of $\beta / a=1$, both Eqs. (9) and (11) give $A=1 / 2$, and the concentration gradient in the fluid resulting from Eq. (8) equals the constant imposed value everywhere.

With knowledge of the solution for the solute concentration distribution, we can now proceed to find the flow field in a cell. The fluid surrounding the cylinder is assumed to be incompressible and Newtonian. Due to the low Reynolds number, the diffusioosmotic motion of the fluid outside the thin diffuse layer is governed by the steady twodimensional differential equation for creeping flows,

$\nabla^{2}\left(\nabla^{2} \Psi\right)=0$,

where $\Psi(\rho, \phi)$ is the stream function related to the $\rho$ and $\phi$ components of the velocity field by

$v_{\rho}=\frac{1}{\rho} \frac{\partial \Psi}{\partial \phi}$,

$v_{\phi}=-\frac{\partial \Psi}{\partial \rho}$.

The boundary condition for the fluid velocity at the surface of the cylinder is $[2,29]$

$\rho=a: \quad v_{\rho}=0$,

$v_{\phi}=-\frac{k T}{\eta} L^{*} K \frac{\partial n}{\rho \partial \phi}$.

The apparent slip velocity given by Eq. (14b) results from Eq. (1) for the local diffusioosmosis caused by the cylinder-solute interaction energy $\Phi(y)$ and the tangential gradient $\partial n / \rho \partial \phi$ along the cylinder surface, which can be evaluated from the solute concentration distribution given by Eq. (8). On the outer boundary of the cell, the Happel model $[8,9]$ assumes that the radial velocity relative to the bulk flow and the shear stress are zero; viz., 
$\rho=b: \quad v_{\rho}=-U_{\mathrm{t}} \cos \phi$,

$\tau_{\rho \phi}=\eta\left[\rho \frac{\partial}{\partial \rho}\left(\frac{v_{\phi}}{\rho}\right)+\frac{1}{\rho} \frac{\partial v_{\rho}}{\partial \phi}\right]=0$

where $U_{\mathrm{t}}$ is the transverse diffusioosmotic velocity of the bulk fluid to be determined.

A solution to Eq. (12) suitable for satisfying boundary conditions on the cylindrical surfaces is [30]

$\Psi=\left(C \rho^{-1}+D \rho \ln \frac{\rho}{a}+E \rho+F \rho^{3}\right) \sin \phi$,

where the constants $C, D, E$, and $F$ are to be determined from Eqs. (14a), (14b), (15a) and (15b) using Eqs. (8), (13a) and (13b). The procedure is straightforward, with the result

$C=a^{2} \omega\left[U_{\mathrm{t}}+V A \ln \varphi\right]$,

$D=2 \omega\left[U_{\mathrm{t}}\left(1+\varphi^{2}\right)-V A\left(1-\varphi^{2}\right)\right]$,

$E=-\omega\left(1-\varphi^{2}\right)\left[U_{\mathrm{t}}+V A \ln \varphi\right]$,

$F=-a^{-2} \omega \varphi^{2}\left[U_{\mathrm{t}}+V A \ln \varphi\right]$,

where

$V=\frac{k T}{\eta} L^{*} K E_{\mathrm{t}}$,

$\omega=\left[1-\varphi^{2}+\left(1+\varphi^{2}\right) \ln \varphi\right]^{-1}$.

With this solution, the components of the fluid velocity can be calculated by using Eqs. (13a) and (13b). Note that the characteristic velocity defined by Eq. (18a) is identical to the diffusioosmotic velocity of the fluid along a planar surface given by Eq. (1).

The drag force (in the $x$ direction) exerted by the fluid on the cylinder per unit length is [30]

$F_{\mathrm{d}}=4 \pi \eta D$.

At the steady state, the net force acting on the cylinder must vanish; viz., $D=0$. With this constraint, Eq. (17b) yields the transverse diffusioosmotic velocity of the bulk fluid,

$U_{\mathrm{t}}=V A\left(1-\varphi^{2}\right)\left(1+\varphi^{2}\right)^{-1}$,

where $A$ is given by Eq. (9) or Eq. (11).

If the Kuwabara model [10] for the boundary conditions of the fluid flow at the virtual surface of the cell, which assumes that the radial velocity relative to the bulk flow and the vorticity are zero, is used, Eq. (15b) is replaced by

$\rho=b: \quad(\nabla \times v)_{z}=\frac{\partial v_{\phi}}{\partial \rho}+\frac{v_{\phi}}{\rho}-\frac{1}{\rho} \frac{\partial v_{\rho}}{\partial \phi}=0$.

With this change, the stream function $\Psi$ can still be expressed in the form of Eq. (16), and the coefficients $C, D, E$, and $F$ should be determined by boundary conditions Eqs. (14a), (14b), (15a) and (21). The result is

$$
\begin{aligned}
C= & a^{2} \omega^{\prime}\left[U_{\mathrm{t}}(2-\varphi)+V A(1-\varphi+2 \ln \varphi)\right], \\
D= & 4 \omega^{\prime}\left[U_{\mathrm{t}}-V A(1-\varphi)\right], \\
E= & -\omega^{\prime}\left[2 U_{\mathrm{t}}(1-\varphi)\right. \\
& \left.+V A\left(1-\varphi^{2}+2 \ln \varphi\right)\right], \\
F= & -a^{-2} \omega^{\prime} \varphi\left[U_{\mathrm{t}}-V A(1-\varphi)\right],
\end{aligned}
$$

where

$\omega^{\prime}=\left(3-4 \varphi+\varphi^{2}+2 \ln \varphi\right)^{-1}$.

The fact that there is no drag force exerted on the cylinder requires $D=0$, and Eq. (22b) gives the bulk transverse diffusioosmotic velocity as

$U_{\mathrm{t}}=V A(1-\varphi)$.

For any combination of $\beta / a$ and $\varphi$, the Happel model always results in a slightly higher value of $U_{\mathrm{t}}$ than the Kuwabara model does (by a factor $\left.(1+\varphi)\left(1+\varphi^{2}\right)^{-1}\right)$, which occurs because the zerovorticity boundary condition yields a larger energy dissipation in the cell than that due to the drag on the cylinder alone. Note that the Happel model has an advantage in that it does not require an exchange of mechanical energy between the cell and the environment [30].

For the diffusioosmosis driven by the longitudinal component $E_{\mathrm{l}} \mathbf{e}_{z}$ of the solute concentration gradient, there is no polarization of the thin diffuse layers or disturbance in the fluid velocity and solute concentration fields caused by the curvature of the cylinders like the above analysis for the transverse motion. The longitudinal diffusioosmotic velocity of the fluid solution is given by Eq. (1), and the bulk fluid velocity (in the direction opposite to $E_{1} \mathbf{e}_{z}$ ) through the ordered array of cylinders can be written as

$U_{1}=\frac{k T}{\eta} L^{*} K E_{1}$. 
The overall diffusioosmotic velocity of the bulk fluid caused by an arbitrarily oriented solute gradient is the vectorial sum of the transversal and longitudinal contributions,

$U=-\left(U_{\mathrm{t}} \mathbf{e}_{x}+U_{1} \mathbf{e}_{z}\right)$.

Thus, in general, the direction of the bulk diffusioosmotic flow will not be parallel to the prescribed solute gradient.

\section{Results and discussion}

Due to the differences in the boundary conditions for the solute concentration and fluid velocity distributions at the virtual surface $\rho=b$ of the unit cell, four cases of the cell model can be defined:

Case I: The Happel model with a specified concentration gradient at the virtual surface (the boundary conditions at $\rho=b$ are described by Eqs. (7), (15a) and (15b));

Case II: The Happel model with a specified concentration profile at the virtual surface (the boundary conditions at $\rho=b$ are described by Eqs. (10), (15a) and (15b));

Case III: The Kuwabara model with a specified concentration gradient at the virtual surface (the boundary conditions at $\rho=b$ are described by Eqs. (7), (15a) and (21));

Case IV: The Kuwabara model with a specified concentration profile at the virtual surface (the boundary conditions at $\rho=b$ are described by Eqs. (10), (15a) and (21)).

The analytical solutions of the concentration and flow fields in the unit cell and the diffusioosmotic velocity of the bulk fluid have been obtained in the previous section for all of the four cases.

In Case I, the transverse diffusioosmotic velocity is given by Eq. (20) with coefficient $A$ defined by Eq. (9). This velocity can be expressed as

$$
\begin{aligned}
U_{\mathrm{t}}= & U_{t}^{(0)}\left(1+\frac{\beta}{a}\right)\left[1+\frac{\beta}{a}-\left(1-\frac{\beta}{a}\right) \varphi\right]^{-1}\left(1-\varphi^{2}\right) \\
& \times\left(1+\varphi^{2}\right)^{-1}
\end{aligned}
$$

where

$$
U_{t}^{(0)}=\left(1+\frac{\beta}{a}\right)^{-1} \frac{k T}{\eta} L^{*} K E_{\mathrm{t}},
$$

which is the transverse diffusioosmotic velocity of the bulk fluid in the limit $\varphi=0$. Note that Eq. (28) is also the diffusiophoretic velocity of an isolated circular cylindrical particle with a thin but polarized diffuse layer caused by the transversely imposed solute concentration gradient [29].

In Case II, the transverse diffusioosmotic velocity of the bulk fluid can be evaluated by Eq. (20) with $A$ given by Eq. (11), and its expression parallel to Eq. (27) for Case I is

$$
\begin{aligned}
U_{\mathrm{t}}= & U_{t}^{(0)}\left(1+\frac{\beta}{a}\right)\left[1+\frac{\beta}{a}+\left(1-\frac{\beta}{a}\right) \varphi\right]^{-1}\left(1-\varphi^{2}\right) \\
& \times\left(1+\varphi^{2}\right)^{-1} .
\end{aligned}
$$

In Case III, the transverse diffusioosmotic velocity of the fluid can be obtained by the substitution of Eq. (9) into Eq. (24), with the result

$U_{\mathrm{t}}=U_{\mathrm{t}}^{(0)}\left(1+\frac{\beta}{a}\right)\left[1+\frac{\beta}{a}-\left(1-\frac{\beta}{a}\right) \varphi\right]^{-1}(1-\varphi)$.

In Case IV, the transverse diffusioosmotic velocity is given by Eq. (24) with $A$ defined by Eq. (11), and it can be expressed as

$U_{\mathrm{t}}=U_{t}^{(0)}\left(1+\frac{\beta}{a}\right)\left[1+\frac{\beta}{a}+\left(1-\frac{\beta}{a}\right) \varphi\right]^{-1}(1-\varphi)$.

It can be found from Eqs. (27)-(31) that the transverse diffusioosmotic velocity of the fluid solution within an ordered array of identical cylinders predicted by the cell model is quite sensitive to the boundary conditions specified at the virtual surface of the cell. The boundary condition for the solute concentration at the virtual surface $\rho=b$ determines the dependence of the normalized diffusioosmotic velocity $U_{t} / U_{t}^{(0)}$ as a function of $\beta / a$, while the boundary condition for the fluid velocity at $\rho=b$ controls the connection of $U_{\mathrm{t}} / U_{\mathrm{t}}^{(0)}$ with the remainder part.

The normalized transverse diffusioosmotic velocity of the bulk fluid in the fiber matrix, $U_{\mathrm{t}} / U_{\mathrm{t}}^{(0)}$, 
as calculated from Eqs. (27)-(31) for the four cases of the cell model, is plotted versus the volume fraction of the cylinders, $\varphi$, in Figs. 2-4 for various values of the parameter $\beta / a$. In all cases, $U_{\mathrm{t}} / U_{\mathrm{t}}^{(0)}=1$ in the limit $\varphi=0$. The calculations are presented up to $\varphi=0.9$, which corresponds to the maximum attainable volume fraction for a swarm of identical parallel cylinders (triangularly ordered, [31]).

In Fig. 2, the normalized diffusioosmotic velocity $U_{\mathrm{t}} / U_{\mathrm{t}}^{(0)}$ of the fluid within an array of identical circular cylinders with $\beta / a=1$ is plotted as a function of $\varphi$ for the Happel cell model (Cases I and II, which are the same with $\beta / a=1)$ and for the Kuwabara cell model (Cases III and IV, which are also the same with $\beta / a=1)$. It is clearly shown that the Kuwabara model predicts a somewhat smaller diffusioosmotic velocity than the Happel model does.

Fig. 3(a) shows the results of the normalized diffusioosmotic velocity $U_{\mathrm{t}} / U_{\mathrm{t}}^{(0)}$ within the array of circular cylinders as a function of $\varphi$ for the limiting case of $\beta / a=0$. It can be seen that, under this situation, $U_{\mathrm{t}} / U_{\mathrm{t}}^{(0)}$ is always greater than unity (and has a maximum at some value of $\varphi$ ) for Case $\mathrm{I}$, is a monotonically decreasing function of $\varphi$ for

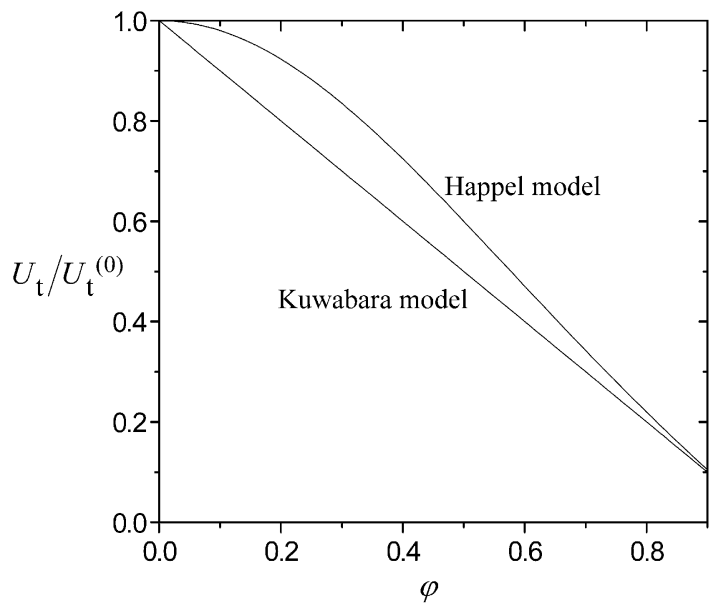

Fig. 2. Plots of the normalized transverse diffusioosmotic velocity of a fluid solution in an ordered array of identical circular cylinders with $\beta / a=1$ versus the volume fraction of the cylinders for the Happel cell model (Cases I and II, Eq. (27) or Eq. (29)) and the Kuwabara cell model (Cases III and IV, Eq. (30) or Eq. (31)).

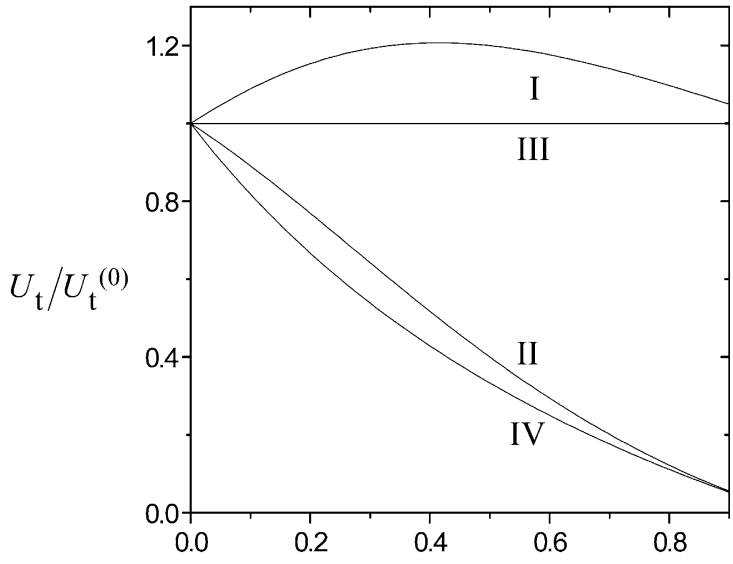

(a)

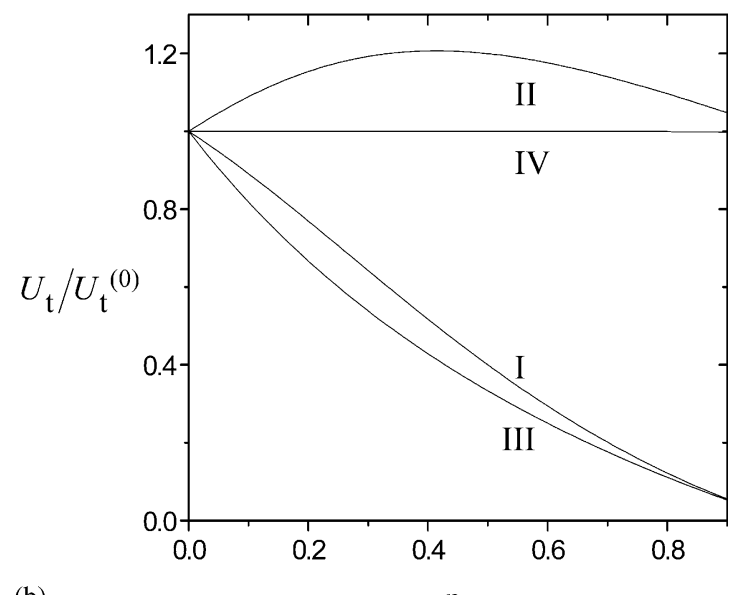

(b)

$\varphi$

Fig. 3. Plots of the normalized transverse diffusioosmotic velocity of a fluid solution in an ordered array of identical circular cylinders versus the volume fraction of the cylinders: (a) $\beta / a=0$; (b) $\beta / a \rightarrow \infty$. The curves with labels I, II, III, and IV represent the cell model calculations from Eqs. (27), (29)-(31), respectively.

Cases II and IV, and is independent of $\varphi$ for Case III. The results of $U_{\mathrm{t}} / U_{\mathrm{t}}^{(0)}$ within the array of cylinders as a function of $\varphi$ for the other limiting case of $\beta / a \rightarrow \infty$ are plotted in Fig. 3(b). Now, $U_{\mathrm{t}} /$ $U_{\mathrm{t}}^{(0)}$ is greater than unity at all values of $\varphi$ (and has a maximum) for Case II, is a monotonically decreasing function of $\varphi$ for Cases I and III, and is independent of $\varphi$ for Case IV. It can be found from Eqs. (27)-(31) that, for the situations with $\beta$ / $a>1$, the transverse diffusioosmotic velocity of the bulk fluid predicted by Cases I and III is 


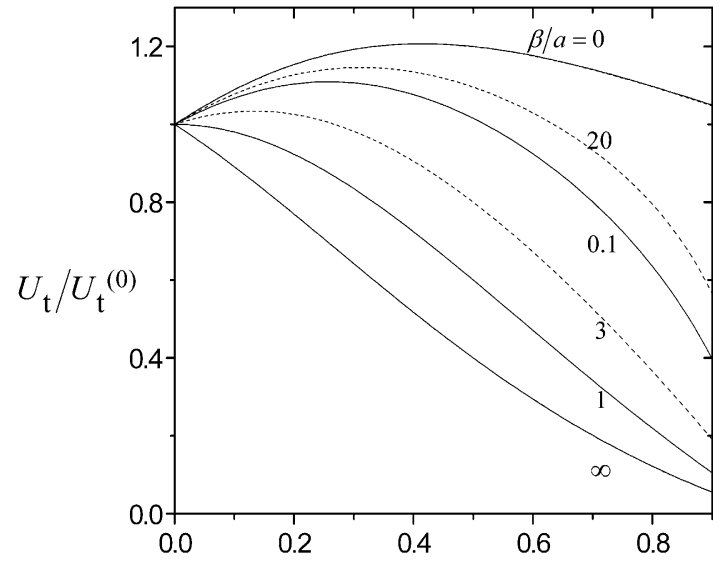

(a)

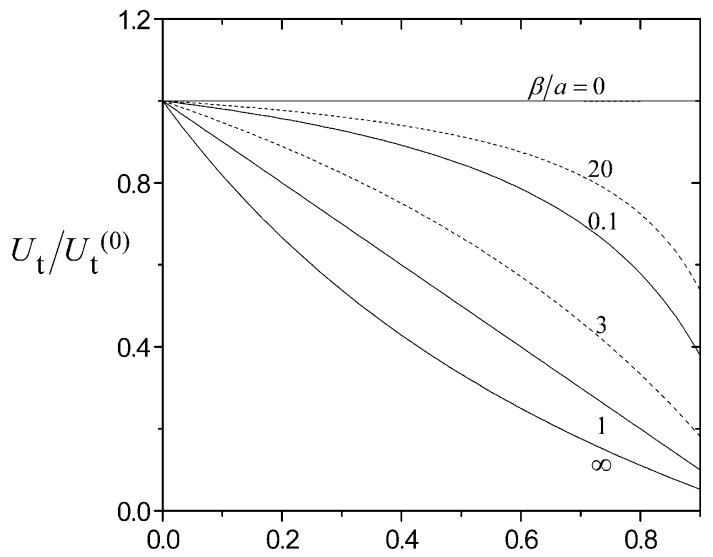

(b)

$$
\varphi
$$

Fig. 4. Plots of the normalized transverse diffusioosmotic velocity of a fluid solution in an ordered array of identical circular cylinders versus the volume fraction of the cylinders with $\beta / a$ as a parameter: (a) Case I (Eq. (27), solid curves) and Case II (Eq. (29), dashed curves); (b) Case III (Eq. (30), solid curves) and Case IV (Eq. (31), dashed curves). The dashed curves of $\beta / a \rightarrow \infty$ and $\beta / a=0$ coincide with the solid curves of $\beta / a=0$ and $\beta / a \rightarrow \infty$, respectively.

smaller than that predicted by Cases II and IV, respectively (as illustrated in Fig. 3(b)), which is opposite to the outcome for the situations with $\beta$ / $a<1$ (as illustrated in Fig. 3(a)).

In Fig. 4, the normalized diffusioosmotic velocity $U_{\mathrm{t}} / U_{\mathrm{t}}^{(0)}$ of the fluid within the array of circular cylinders is plotted as a function of $\varphi$ for the four cases of the cell model with $\beta / a$ as a parameter. For a fixed value of $\varphi, U_{\mathrm{t}} / U_{\mathrm{t}}^{(0)}$ decreases monotonically with an increase in $\beta / a$ in Cases I and III, but is a monotonically increasing function of $\beta / a$ in Cases II and IV. For Case I with $\beta / a<1$ or Case II with $\beta / a>1, U_{\mathrm{t}} / U_{\mathrm{t}}^{(0)}$ increases with an increase in $\varphi$ in a dilute suspension, while this normalized velocity is a monotonically decreasing function of $\varphi$ for Case I with $\beta / a \geq 1$ or for Case II with $\beta / a \leq 1$. For the special situations of Case III with $\beta / a=0$ and Case IV with $\beta / a \rightarrow \infty, U_{\mathrm{t}} /$ $U_{\mathrm{t}}^{(0)}$ is independent of $\varphi$; however, this normalized velocity decreases monotonically with an increase in $\varphi$ in all the other situations of Cases III and IV.

\section{Concluding remarks}

In this paper, the steady-state diffusioosmosis of a fluid solution of an uncharged solute with a uniformly imposed and arbitrarily oriented concentration gradient in an ordered array of identical circular cylinders with thin but polarized interfacial layers is analyzed using the unit cell model with various boundary conditions at the virtual surface of the cell. On the basis of the assumption of small Peclet and Reynolds numbers, the solute concentration and fluid flow fields in the cell were solved analytically and the diffusioosmotic velocity of the bulk fluid as a function of the porosity of the array of cylinders was obtained in closedform expressions given by Eqs. (27)-(31). Comparisons of the results of the cell model with different conditions at the outer boundary of the cell have also been provided.

We note that the four cases of the cell model defined in the previous section lead to somewhat different results for the diffusioosmotic velocity of the fluid. The unit cell models with various boundary conditions at the virtual surface of the cell have also been used in the literature to study the electrophoresis [14-17,20] and diffusiophoresis [26] of suspensions of colloidal spheres with thin diffuse layers. The results of those studies indicate that the tendency of the dependence of the normalized particle mobility on the volume fraction of the particles in Cases I and III is not correct in comparison with the ensemble-averaged results obtained by using the concept of statistical mechanics. So, the boundary condition represented by Eq. (7) might not be as accurate as that 
represented by Eq. (10), probably due to the fact that the angular component of the solute concentration gradient at the virtual surface of the cell is not specified in Eq. (7). It was also shown that the electrophoretic and diffusiophoretic velocities predicted by Case IV of the cell model agree quite well with those obtained from the statistical model $[32,33]$ and from an experimental study [34] for dilute suspensions of particles. A possible reason for this outcome might be the fact that the Kuwabara boundary condition of zero vorticity is consistent with the irrotational-flow environment generated by an electrophoretic or diffusiophoretic particle with a thin diffuse layer. For the system of diffusioosmosis in a fibrous porous medium discussed in the present work, the relevant experimental data, which are not available yet, would be needed to confirm the validity of each case of the cell model at various ranges of $\beta / a$ and $\varphi$.

Eq. (28) for the limiting case of $\varphi=0$ can be used to express the diffusiophoretic velocity of a single circular cylindrical particle in the direction normal to its axis. As to the diffusiophoretic motion of a cylinder generated by a longitudinal solute concentration gradient, there is no disturbance in the solute concentration and fluid velocity fields caused by the curvature of the cylinder. Thus, the longitudinal velocity of the cylinder is given by Eq. (25). For the diffusiophoresis of a circular cylindrical particle oriented arbitrarily with respect to the imposed concentration gradient, the particle velocity is the vectorial sum of its transversal and longitudinal contributions. For an ensemble of circular cylinders with random orientation, the average diffusiophoretic velocity (aligned with the direction of the applied concentration gradient $\left.\nabla n_{\infty}\right)$ can be obtained by two thirds of the value given by Eq. (28) (with $E_{\mathrm{t}}=$ $\left.\left|\nabla n_{\infty}\right|\right)$ plus one third of the value given by Eq. (25) (with $E_{\mathrm{t}}=\left|\nabla n_{\infty}\right|$ ) [29].

\section{Acknowledgements}

This work was supported by the National Science Council of the Republic of China under Grant NSC90-2214-E-002-019.

\section{References}

[1] S.S. Dukhin, B.V. Derjaguin, in: E. Matijevic (Ed.), Surface and Colloid Science, vol. 7, Wiley, New York, 1974.

[2] J.L. Anderson, M.E. Lowell, D.C. Prieve, J. Fluid Mech. 117 (1982) 107.

[3] D.C. Prieve, J.L. Anderson, J. Ebel, M.E. Lowell, J. Fluid Mech. 148 (1984) 247

[4] J.C. Fair, J.F. Osterle, J. Chem. Phys. 54 (1971) 3307.

[5] V. Sasidhar, E. Ruckenstein, J. Colloid Interface Sci. 85 (1982) 332.

[6] G.B. Westermann-Clark, J.L. Anderson, J. Electrochem. Soc. 130 (1983) 839.

[7] H.J. Keh, J.H. Wu, Langmuir 17 (2001) 4216.

[8] J. Happel, AIChE J. 4 (1958) 197.

[9] J. Happel, AIChE J. 5 (1959) 174.

[10] S. Kuwabara, J. Phys. Soc. Japan 14 (1959) 527.

[11] S. Levine, G.H. Neale, N. Epstein, J. Colloid Interface Sci. 57 (1976) 424.

[12] H.J. Keh, J.M. Ding, J. Colloid Interface Sci. 227 (2000) 540.

[13] F. Carrique, F.J. Arroyo, A.V. Delgado, Colloids Surf. A 195 (2001) 157.

[14] S. Levine, G.H. Neale, J. Colloid Interface Sci. 47 (1974) 520.

[15] V.N. Shilov, N.I. Zharkikh, Yu.B. Borkovskaya, Colloid J. USSR (English Translation) 43 (1981) 434.

[16] N.I. Zharkikh, V.N. Shilov, Colloid J. USSR (English Translation) 43 (1982) 865.

[17] M.W. Kozak, E.J. Davis, J. Colloid Interface Sci. 127 (1989) 497.

[18] H. Ohshima, J. Colloid Interface Sci. 188 (1997) 481.

[19] T.J. Johnson, E.J. Davis, J. Colloid Interface Sci. 215 (1999) 397.

[20] Y.K. Wei, H.J. Keh, Langmuir 17 (2001) 1437.

[21] F. Carrique, F.J. Arroyo, A.V. Delgado, J. Colloid Interface Sci. 243 (2001) 351.

[22] N.I. Zharkikh, Yu.B. Borkovskaya, Colloid J. USSR (English Translation) 43 (1982) 520.

[23] M.W. Kozak, E.J. Davis, J. Colloid Interface Sci. 112 (1986) 403.

[24] H. Ohshima, J. Colloid Interface Sci. 210 (1999) 397.

[25] E. Lee, Y. Lee, F. Yen, J. Hsu, J. Colloid Interface Sci. 223 (2000) 223.

[26] H.J. Keh, Y.K. Wei, Colloid Polym. Sci. 270 (2000) 539.

[27] R.W. O'Brien, L.R. White, J. Chem. Soc. Faraday Trans. II 74 (1978) 1607.

[28] J.L. Anderson, D.C. Prieve, Langmuir 7 (1991) 403.

[29] H.J. Keh, S.B. Chen, Langmuir 9 (1993) 1142.

[30] J. Happel, H. Brenner, Low Reynolds Number Hydrodynamics, Martinus Nijhoff, The Netherlands, 1983.

[31] J.G. Berryman, Phys. Rev. A 27 (1983) 1053.

[32] H.J. Keh, S.C. Luo, Phys. Fluids 7 (1995) 2122.

[33] H.J. Tu, H.J. Keh, J. Colloid Interface Sci. 231 (2000) 265.

[34] C.F. Zukoski, D.A. Saville, J. Colloid Interface Sci. 115 (1987) 422. 